UCSD/PTH 01-11

\title{
Bulk Observers in Non-Factorizable Geometries
}

\author{
Benjamín Grinstein* and Detlef R. Nolte ${ }^{\dagger}$ \\ *Department of Physics, University of California at San Diego, La Jolla, CA 92093 \\ ${ }^{\dagger}$ Institute for Advanced Study, Princeton, NJ 08540
}

(June 18, 2001)

\begin{abstract}
We consider five dimensional non-factorizable geometries where the transverse dimension is bounded and the remaining (parallel) dimensions are not. We study the construction of effective theories at distances much longer than the transverse size. An observer unable to resolve the transverse direction can only measure distances along the parallel dimensions, but the non-factorizable geometry makes the length of a curve along the parallel dimension sensitive to where on the transverse direction the curve lies. We show that long geodesics that differ in their endpoints only by shifts along the transverse direction all have the same length to within the observer's resolution. We argue that this is the correct notion of distance in the effective theory for a bulk observer. This allows us to present a consistent interpretation of what is measured by observers that live either on a brane or in the bulk.
\end{abstract}

PACS: $11.10 . \mathrm{Kk}, 04.50 .+\mathrm{h}$ 
Introduction and Conclusions. Randall and Sundrum have proposed a solution to the hierarchy problem based on a $Z_{2}$ five-dimensional orbifold with 3-branes at the fixed points [1]. There is a negative cosmological constant $\Lambda$ and tension on the branes, $V_{\text {hid }}$ and $V_{\text {vis }}$. Then the metric

$$
d s^{2}=G_{M N} d x^{M} d x^{N}=a^{2}(y) \eta_{\mu \nu} d x^{\mu} d x^{\nu}-d y^{2}
$$

solves Einstein's equations provided $a(y)=e^{-k|y|}$ and

$$
V_{\text {hid }}=-V_{v i s}=24 M^{3} k \quad \Lambda=-24 M^{3} k^{2}
$$

where $M$ is the fundamental 5 -dimensional gravitational mass scale. The brane with negative tension, $V_{v i s}$, contains the visible universe and is located at $y=y_{c}$ while the hidden brane, with tension $V_{h i d}$, is at $y=0$. We will refer to this as the RS model, to the $y$-coordinate as the transverse direction and to the remaining ones as the parallel directions, since they are parallel to the bounding branes.

In situations of physical interest the transverse direction is microscopic. Macroscopic observers cannot resolve lengths as small as the transverse dimension. Effectively they see a world of four dimensions. Naively one would attempt to describe physics at long distances by ignoring the transverse direction. This is, after all, what is commonly done to infer the spectrum of Kaluza-Klein excitations in factorizable geometries. But for non-factorizable geometries the invariant interval of a line between the points $\left(0, y_{1}\right)$ and $\left(\Delta x, y_{2}\right)$ depends on the transverse direction $y$. This dependence can give results for the length that vary up to a factor of $\exp \left(k y_{c}\right)$. The macroscopic observer is, however, unable to discern the value of $y$. We are faced with the question: between two points 0 and $\Delta x$ what is the length measured by the macroscopic observer? Many physical questions require an answer to this. For example, what does the argument of a field, $\phi(x)$, in an effective theory represent? Since mass is the inverse length for exponential fall-off of a static field, what is the mass of a field? Although much of the physics of these models has been understood [4], most of the research has been focused on the construction of effective field theories for brane observers and not 
bulk observers. For an observer restricted to a brane the answer is well known. Distances measured by an apparatus made solely of components restricted to a brane correspond to distances inferred by the induced metric on the brane. The RS model has been analyzed in [7], [8] by using only the induced metric. But an observer living in the bulk will be spread out over the microscopic transverse direction and it is not obvious which 4 - dim metric will give the correct physical distances. Our main result is as follows: distances measured by an apparatus made of unrestricted components (bulk fields) correspond to distances measured on the visible brane, provided these distances are much larger than the brane separation. Specifically, the distance between points $(0, y)$ and $\left(\Delta x, y^{\prime}\right)$ is, to high accuracy, $\exp \left(-k y_{c}\right) \Delta x$ for any $y$ and $y^{\prime}$, provided $\exp \left(-k y_{c}\right) \Delta x \gg y_{c}$.

Effective theories for brane observers at long distances have been constructed by "integrating-out" the transverse direction. Let us review the modification by Ozeki and Shimoyama [8] of the computation of Goldberger and Wise [2] for the case of a scalar field in the background of the geometry in Eq. (1). The action

$$
S=\frac{1}{2} \int d^{4} x d y \sqrt{G}\left(G^{A B} \partial_{A} \Phi \partial_{B} \Phi-M^{2} \Phi^{2}\right)
$$

admits a decomposition is in terms of the modes

$$
\Phi(x, y)=\sum_{n} \psi_{n}(x) \xi_{n}(y)
$$

normalized so that the effective action is written in terms of the induced metric $g_{\text {ind }}$ according to $[8]$

$$
\int_{-y_{c}}^{y_{c}} d y e^{-2 k|y|} \xi_{n}(y) \xi_{m}(y)= \begin{cases}e^{-2 k\left|y_{c}\right|} \delta_{n m} & \text { observer at } y_{c} \\ \delta_{n m} & \text { observer at } y_{0}\end{cases}
$$

and which satisfy

$$
-\frac{d}{d y}\left(e^{-4 k|y|} \frac{d \xi_{n}}{d y}\right)+M^{2} e^{-4 k|y|} \xi_{n}=m_{n}^{2} e^{-2 k|y|} \xi_{n},
$$

giving 


$$
S=\frac{1}{2} \sum_{n} \int d^{4} x \sqrt{g_{\text {ind }}}\left[g_{i n d}^{\mu \nu} \partial_{\mu} \psi_{n} \partial_{\nu} \psi_{n}-m_{n}^{2} \psi_{n}^{2}\right] .
$$

Analysis of the solutions of these equations give the correct mass parameters for brane observers. However, it is not obvious how to apply this procedure to a bulk observer. Because of the finite transverse width of the bulk observer, the notion of an induced metric is not well defined. We argue that in the RS model distances measured by a bulk observer are equal to distances measured by an observer on the visible brane up to errors of order of the length of the extra dimension. Therefore, we suggest that the KK mass spectrum as seen by a bulk observer is also equal to the spectrum seen by an observer on the visible brane.

Brane Observers and Bulk Observers. Effective theories at long distances are meant to accurately describe the world as seen by an observer who has limited resolution. In a non-factorizable geometry we must make a distinction between observers that live on one brane or the other and observers that live in the bulk. We will refer to observers whose measuring devices are constructed solely of fields and particles constrained to a brane as "brane observers," and to those whose measuring devices are built of unconstrained fields and particles as "bulk observers." As we will see there are some qualitative and quantitative differences in the observations they make, so the distinction is important.

We imagine that either observer can make a construction, following Einstein, of locally inertial frames with identical meter sticks and synchronized clocks. To better understand the construction it will be necessary to know how to describe freely falling observers and how observers measure distances and time. The former entails finding geodesics while the latter requires finding the minimum distance between points on a fixed time hypersurface, that is, geodesics of spatial sections.

We write the geodesic equation in the RS model for $0<y<y_{c}$, and the case of $-y_{c}<$ $y<0$ can be obtained formally by replacing $k \rightarrow-k$ :

$$
\frac{d^{2} x^{\mu}}{d \tau^{2}}-2 k \frac{d y}{d \tau} \frac{d x^{\mu}}{d \tau}=0
$$




$$
\frac{d^{2} y}{d \tau^{2}}-k e^{-2 k y} \eta_{\mu \nu} \frac{d x^{\mu}}{d \tau} \frac{d x^{\nu}}{d \tau}=0
$$

For $\eta_{\mu \nu} \frac{d x^{\mu}}{d \tau} \frac{d x^{\nu}}{d \tau}>0$ time-like geodesics are [6]

$$
\begin{aligned}
x^{\mu} & =x_{0}^{\mu}+\frac{v_{0}^{\mu}}{k v_{0}^{2}} \tan (k \tau) \\
y & =-\frac{1}{2 k} \ln \left(v_{0}^{2} \cos ^{2}(k \tau)\right),
\end{aligned}
$$

where $v_{0}^{2} \equiv \eta_{\mu \nu} v_{0}^{\mu} v_{0}^{\nu}$. The constants of integration are $v_{0}^{\mu}$ and $x_{0}^{\mu}$, and they satisfy $v_{0}^{2}>0$. One can also shift $\tau \rightarrow \tau-\tau_{0}$, but we have chosen $\tau_{0}=0$ so that $d y / d \tau=0$ at $\tau=0$, for convenience. The solution for $y=y(\tau)$ should be interpreted bearing in mind the periodicity and identification conditions that define the orbifold. Eq. (8) implies that at the brane $y=y_{c}$ the velocity $d y / d \tau$ is continuous. Using the reflection symmetry and periodicity this implies that, if we insist in describing the motion on the interval $\left[0, y_{c}\right]$, the particle bounces off the brane at $y=y_{c}$ reversing its velocity $d y / d \tau$. The motion $y(\tau)$ is periodic, with period $\frac{2}{k} \cos ^{-1}\left(e^{-k y_{c}} / \sqrt{v_{0}^{2}}\right)$.

In terms of the coordinate time $t=x^{0}$, these solutions give the expected straight line uniform motion in the large three spatial dimensions:

$$
\begin{aligned}
x^{i} & =x_{0}^{i}+\frac{v_{0}^{i}}{v_{0}^{0}}\left(t-t_{0}\right) \\
y & =\frac{1}{2 k} \ln \left(\frac{1}{v_{0}^{2}}+\frac{v_{0}^{2}}{v_{0}^{02}} k^{2}\left(t-t_{0}\right)^{2}\right) .
\end{aligned}
$$

As expected for motion in a gravitational field, the particle necessarily has non-vanishing velocity (except at $\tau=0$ ) and acceleration along the transverse (fifth) dimension. We see that a classical observer is either extended out from $y=y_{c}$ into the bulk, or is, at best, in a limiting sense, constrained to $y=y_{c}$.

For completeness we give the case of massless particles too. If $\eta_{\mu \nu} \frac{d x^{\mu}}{d \tau} \frac{d x^{\nu}}{d \tau}=0$ then $d y / d \tau$ must be constant. But then $d s^{2} / d \tau^{2} \geq 0$ only if $d y / d \tau=0$. So this describes only massless particles (light-like geodesics). This is consistent with the observation that the Klein-Gordon equation in the warped background admits $y$-independent solutions only for massless particles. Light-like geodesics are given, for $\eta_{\mu \nu} \frac{d x^{\mu}}{d \tau} \frac{d x^{\nu}}{d \tau}>0$, by 


$$
\begin{aligned}
x^{\mu} & =x_{0}^{\mu}+\frac{v_{0}^{\mu}}{k^{2} v_{0}^{2} \tau} \\
y & =-\frac{1}{k} \ln \left( \pm k \sqrt{v_{0}^{2}} \tau\right) .
\end{aligned}
$$

What is the physical distance between points on this space? Consider two points on a fixed time hypersurface, say $t=0$, separated by some large coordinate distance $\Delta x$. It would seem that the physical distance depends on the transverse coordinate $y$ of these points. If so, how can one build an effective four dimensional theory at physical distances much larger than $y_{c}$ ? The answer, as we now show, is that at large $\Delta x$, the physical distance these points is $\exp \left(-k y_{c}\right) \Delta x$, independent of the $y$-coordinates of these points. The correction to this statement is of order $y_{c}$, so an observer without the resolution to observe the fifth dimension is also oblivious to this correction.

On a fixed $t$ hypersurface the geodesic equations are

$$
\begin{aligned}
\frac{d^{2} x^{i}}{d \tau^{2}}-2 k \frac{d y}{d \tau} \frac{d x^{i}}{d \tau} & =0 \\
\frac{d^{2} y}{d \tau^{2}}+k e^{-2 k y} \delta_{i j} \frac{d x^{i}}{d \tau} \frac{d x^{j}}{d \tau} & =0
\end{aligned}
$$

with solutions

$$
\begin{aligned}
x^{i}(\tau) & =x_{0}^{i}+\frac{\beta^{i}}{k^{2} \beta^{2}} \tanh \left[\omega\left(\tau-\tau_{0}\right)\right] \\
y(\tau) & =-\frac{1}{k} \ln \left(k \beta \cosh \left[\omega\left(\tau-\tau_{0}\right)\right]\right),
\end{aligned}
$$

where $\beta \equiv \sqrt{\delta_{i j} \beta^{i} \beta^{j}}$ and $\tau$ is an affine parameter, $\tau \in[0,1]$. The parameter $\omega$ is simply the physical length $\ell$ in units of $k$ along the geodesic, $\omega=k \ell$. Given initial and final points, the parameters of the geodesic are determined by

$$
\begin{aligned}
k \beta & =\frac{\left|\tanh \left[\omega\left(1-\tau_{0}\right)\right]+\tanh \left[\omega \tau_{0}\right]\right|}{k|\Delta x|}, \\
\omega\left(1-\tau_{0}\right) & =\operatorname{arcsinh}\left(\frac{d_{1}^{2}-1+d_{1}^{2} / d_{0}^{2}}{2 d_{1}}\right), \\
\omega \tau_{0} & =\operatorname{arcsinh}\left(\frac{d_{0}^{2}-1+d_{0}^{2} / d_{1}^{2}}{2 d_{0}}\right),
\end{aligned}
$$

where $|\Delta x|^{2} \equiv \delta_{i j}\left(x^{i}(1)-x^{i}(0)\right)\left(x^{j}(1)-x^{j}(0)\right)$ and the quantities $d_{0,1}$ are simply the lengths in units of $k$ between $x^{i}(0)$ and $x^{i}(1)$ along curves of fixed $y$, 


$$
\begin{aligned}
& d_{0}=k|\Delta x| e^{-k y(0)}, \\
& d_{1}=k|\Delta x| e^{-k y(1)} .
\end{aligned}
$$

To understand this solution consider the case $y(0) \leq y(1)$ which is described by $\tau_{0} \geq 1 / 2$. If $\tau_{0} \geq 1$ the coordinate $y(\tau)$ increases monotonically from $y(0)$ to $y(1)$, while for $1 / 2 \leq \tau_{0}<1$ the solution $y(\tau)$ increases monotonically from $y(0)$ beyond $y(1)$ (at $\tau=2 \tau_{0}-1$ ) to a maximum at $y\left(\tau_{0}\right)$ and decreases monotonically back to $y(1)$. The transition between these two distinct behaviors occurs at $\tau_{0}=1$, that is, at a critical $x$ separation given by

$$
k|\Delta x|_{\mathrm{crit}} e^{-k y(1)}=\sqrt{1-e^{-2 k(y(1)-y(0))}}
$$

For $|\Delta x|<|\Delta x|_{\text {crit }}$ the solution has $\tau_{0}>1$.

The solutions to the geodesic equation above do not describe the minimum distance path between points in the orbifold of the RS model because it neglects the presence of fixed points. At large separation $|\Delta x|$ the solution above extends to the region $y>y_{c}$. The shortest path is actually along a $\tau_{0}=1$ geodesic from $y(0)$ to $y_{c}$, then along the brane $y=y_{c}$ and finally back from $y_{c}$ to $y(1)$ along a second $\left(\tau_{0}=0\right)$ geodesic. The shortest path between two points has length

$$
\ell_{\text {phys }}=|\Delta x| e^{-k y_{c}}+\left(2 y_{c}-y(0)-y(1)+\ln 4-2\right)+\mathcal{O}\left(e^{-k\left(y_{c}-\min (y(0), y(1))\right.}\right) .
$$

This applies only provided the length along $x$ is large enough that the above geodesic would hit the $y=y_{c}$ brane. The condition for this is, from Eq. (24), that the length measured along the $y=y_{c}$ brane be larger than $1 / k$.

We can now understand what a brane and a bulk observer are, their similarities and differences. First, Eq. (11) tells us that if we ignore the $y$-motion of a particle in the bulk the motion in the transverse space is like that of a free particle in flat space. Bulk observers, or rather their meter sticks, who have resolution smaller than the brane separation $y_{c}$ are presumably much bigger than $y_{c}$ themselves. They are made of many particles distributed over the $y$-direction. A priori one could expect these particles would spread in $x^{i}$ even if 
they have the same initial velocity, but we see this is not the case (a fortunate state of affairs for the observer).

Second, Eq. (25) tells us that the meter stick this bulk observer will use to measure distances between two points does not depend on the $y$-coordinates of these points. The error introduced by ignoring the endpoint of the meter stick is smaller than the distance between the branes, but we already assumed that the resolution of this observer was worse.

Third, Eq. (25) shows that the distance measured by the bulk observer between two points is what a negative tension brane observer (that is, an observer on the $y=y_{c}$ brane) would measure (between the points projected onto the brane). The distance between these points measured by the positive tension brane observer is, however, exponentially larger.

Last, similar conclusions hold for measurement of time, since motion is uniform in $x^{i}(t)$. Observers can measure two units of time by reflecting a pulse of light off a mirror set at the end of a meter stick.

Interpretation Of The Effective Action. The meaning of effective theories with the transverse direction integrated out is now clear. For a definite example consider the bulk scalar action of (3) and the corresponding effective theory (77). Instead of formally integrating out the $y$-direction, we study the response of the bulk scalar to arbitrary sources smeared over the resolution of the detecting apparatus and separated by distances larger than the resolution. The Green function of the Klein-Gordon equation, $\Delta\left(x, y ; x^{\prime}, y^{\prime}\right)$ can be written in terms of the four dimensional Green function for a particle of mass $m_{n}, \Delta^{(4)}\left(x-x^{\prime} ; m_{n}\right)$, as [7]

$$
\begin{aligned}
\Delta\left(x, y ; x^{\prime}, y^{\prime}\right) & =-\sum_{n} \int \frac{d^{4} q}{(2 \pi)^{4}} \frac{e^{i q \cdot\left(x-x^{\prime}\right)}}{q^{2}-m_{n}^{2}} R_{n}\left(y, y^{\prime}\right) \\
& =\sum_{n} \Delta^{(4)}\left(x-x^{\prime} ; m_{n}\right) R_{n}\left(y, y^{\prime}\right) .
\end{aligned}
$$

Since the residue factorizes, $R_{n}\left(y, y^{\prime}\right)=r_{n}(y) r_{n}\left(y^{\prime}\right)$, the full Green function $\Delta\left(x, y ; x^{\prime}, y^{\prime}\right)$ can be obtained in a four dimensional description by coupling sources $j(x)$ and $j^{\prime}(x)$ to the linear combinations $\sum_{n} r_{n}(y) \psi_{n}(x)$ and $\sum_{n} r_{n}\left(y^{\prime}\right) \psi_{n}(x)$, respectively. The spectrum, $m_{n}$, is determined as solutions to 


$$
\tilde{N}_{\nu}\left(m_{n} z_{1}\right) \tilde{J}_{\nu}\left(m_{n} z_{2}\right)-\tilde{J}_{\nu}\left(m_{n} z_{1}\right) \tilde{N}_{\nu}\left(m_{n} z_{2}\right)=0
$$

where $z_{1,2}=e^{k y} /\left.k\right|_{y=0, y_{c}}, \nu=\sqrt{4+m^{2} / k^{2}}$, and we have introduced the shorthand $\tilde{Z}_{\nu}(z)=$ $\left(1-\frac{\nu}{2}\right) Z_{\nu}(z)+\frac{z}{2} Z_{\nu-1}(z)$ for the combinations of Bessel functions of first and second kind, $J_{\nu}$ and $N_{\nu}$. This is precisely the same equation as found by Goldberger and Wise by means of a different method [2], namely, direct diagonalization of the action integral. For small $m$, the low excitation number spectrum has $m_{n} \sim a\left(y_{c}\right) k$. As above, the full Green function can be written as a sum over poles, Eq. (26), where the residues factorize.

By the arguments of the previous section, an observer on the visible (negative tension) brane sees particles of physical mass $m_{n} / a\left(y_{c}\right) \sim k$. Their 'overlap', or wave-function on the visible brane, is given by $r_{n}\left(y_{c}\right)$. Similarly, since long distances in the bulk correspond to long distances on the visible brane, bulk observers also see particles of physical masses and splittings of order $k$. It is only observers on the hidden (positive tension) brane who see exponentially suppressed masses. There is a simple physical interpretation. Hidden brane observers see masses that have climbed up a potential well and are therefore red-shifted precisely by the warp factor.

The effective action for this theory for a bulk observer or for an observable brane observer is just as in Eq. (7), with the subsidiary information that the coordinates $x^{\mu}$ do not measure physical distance, but exponentially large distance. When the coordinates are rescaled so that they actually correspond to physical distance the effective action is of the same form, but the masses are replaced $m_{n} \rightarrow \exp \left(k y_{c}\right) m_{n}$. Interactions can be included. They are computed by considering $n$-point functions. Similarly, the effective action in (7) correctly accounts for the physics seen by the hidden brane observer, since $x^{\mu}$ does correspond to physical distance there.

Generalizations Similar results are obtained for a wide class of non-factorizable geometries. A simple case is that in which a space built on an $S_{2} / Z_{2}$ orbifold,

$$
d s^{2}=e^{2 A(y)} \eta_{\mu \nu} d x^{\mu} d x^{\nu}-d y^{2},
$$


has a warp factor $A$ that is locally a minimum at both fixed points. At large parallel separations the bulk observers see distances as measured along the brane of smaller warp factor.

An interesting case is the metric for a space with a single brane at a fixed point in Anti-de Sitter 5-space $\left(A d S_{5}\right)$ with Anti-de Sitter $\left(A d S_{4}\right)$ sections [9]. The metric is as in Eq. (28), but with $\eta$ replaced by the metric of $A d S_{4}$. The warp factor is

$$
A=\log \left(\cosh \frac{c-|y|}{L} / \cosh \frac{c}{L}\right)
$$

decreasing from the brane to $y=c$ where it has a minimum and then growing again. Fixed- $t$ geodesics at large parallel separation $\Delta x$ go mostly very close to the hypersurface $y=c$. There is no brane located there, still distance scales for a bulk observer are as if measured along a $y=c$ brane. The graviton is localized on the brane at $y=0$ [10. The spectrum of massive excitations of the graviton is observed to be exponentially lighter by a brane observer than by a bulk observer, giving an amusing inverted hierarchy.

Acknowledgments We would like to thank Ken Intriligator, Ira Rothstein and Witold Skiba for discussions. This work is supported by the Department of Energy under contract No. DOE-FG03-97ER40546. 


\section{REFERENCES}

[1] L. Randall and R. Sundrum, Phys. Rev. Lett. 83, 3370 (1999), hep-ph/9905221.

[2] W. D. Goldberger and M. B. Wise, Phys. Rev. D60, 107505 (1999) hep-ph/9907218.

[3] S. B. Giddings, E. Katz and L. Randall, JHEP 0003, 023 (2000) hep-th/0002091.

[4] C. Csaki, J. Erlich and T. J. Hollowood, Phys. Rev. Lett. 84, 5932 (2000) hepth/0002161. D. J. Chung and K. Freese, Phys. Rev. D 61, 023511 (2000) hepph/9906542]. I. Y. Aref'eva, M. G. Ivanov, W. Muck, K. S. Viswanathan and I. V. Volovich, Nucl. Phys. B 590, 273 (2000) hep-th/0004114. J. M. Cline, C. Grojean and G. Servant, Phys. Rev. Lett. 83, 4245 (1999) [hep-ph/9906523. B. Grinstein, D. R. Nolte and W. Skiba, Phys. Rev. D 63, 105016 (2001) hep-th/0012202].

[5] P. Horava and E. Witten, Nucl. Phys. B 475, 94 (1996) hep-th/9603142. P. Horava and E. Witten, Nucl. Phys. B 460, 506 (1996) hep-th/9510209. P. Bowcock, C. Charmousis and R. Gregory, Class. Quant. Grav. 17, 4745 (2000) hep-th/0007177.

[6] W. Muck, K. S. Viswanathan and I. V. Volovich, Phys. Rev. D 62, 105019 (2000) hep-th/0002132.

[7] B. Grinstein, D. R. Nolte and W. Skiba, Phys. Rev. D 63, 105005 (2001) hep th/0012074.

[8] T. Ozeki and N. Shimoyama, Prog. Theor. Phys. 103, 1227 (2000), hep-th/9912276.

[9] O. DeWolfe, D. Z. Freedman, S. S. Gubser and A. Karch, Phys. Rev. D 62, 046008 (2000) hep-th/9909134. N. Kaloper, Phys. Rev. D 60, 123506 (1999) hep-th/9905210. H. B. Kim and H. D. Kim, Phys. Rev. D 61, 064003 (2000) hep-th/9909053. T. Nihei, Phys. Lett. B 465, 81 (1999) hep-ph/9905487.

[10] A. Karch and L. Randall, JHEP 0105, 008 (2001) hep-th/0011156. 